\title{
MOLECULE INDEX
}

Numbers refer to first page of paper. For references to complete lists of detected molecules, see Appendix 1.

${ }^{26} \mathrm{Al}$ 457

$\mathrm{AlCl}$ 281,457

AlF 281,457

Aliphatics 343,357

$\mathrm{Ar}^{+}$ 373

Aromatics 357

Benzene 469

C

$\mathrm{C}^{+}$ $113,129,141,431$

$\mathrm{C}_{2}$ $129,141,389,393,431$

$\mathrm{C}_{2} \mathrm{H}$ 407,441

$\mathrm{C}_{2} \mathrm{H}_{2}$ $75,253,421,457$

$\mathrm{C}_{2} \mathrm{H}_{4}$ $1,31,219,253,271,331,397,441,469$

$\mathrm{C}_{2} \mathrm{H}_{5}$ 205,253

$\mathrm{C}_{2} \mathrm{H}_{5} \mathrm{OH}$ 253

$\mathrm{C}_{2} \mathrm{H}_{5} \mathrm{CN}$ 31,75

$\mathrm{C}_{2} \mathrm{H}_{6}$ 219,253

$\mathrm{C}_{2} \mathrm{O}$ .... 75

$\mathrm{C}_{2} \mathrm{~S}$ 75,457

$\mathrm{C}_{3}$ $281,407,441$

$\mathrm{C}_{3} \mathrm{H}_{2}$

$\mathrm{C}_{3} \mathrm{H}$ $103,173,253,421,457,477$

$\mathrm{C}_{3} \mathrm{O}$ 457

$\mathrm{C}_{3} \mathrm{~S}$ 75

$\mathrm{C}_{4} \mathrm{H}$ 75,457

$\mathrm{C}_{4} \mathrm{Si}$ 457

$\mathrm{C}_{5}$ 457

$\mathrm{C}_{5} \mathrm{H}$ 281

$\mathrm{C}_{6} \mathrm{H}$ 457

$\mathrm{C}_{8} \mathrm{H}$ 457

$\mathrm{C}_{11} \mathrm{H}$ 1,457

$\mathrm{C}_{60}$ ..1

$\mathrm{C}_{70}$ 281

$\mathrm{C}_{n}$ 281

$\mathrm{C}_{n}$ 287

$\mathrm{C}_{n} \mathrm{H}$ 287

$\mathrm{CaF}$ 287

Carbides 457

CCN 343

$\mathrm{CH}$ 271 $\mathrm{CH}^{+}$ $13,163,253,407,431,441$ $\mathrm{CH}_{2}$ $1,141,407,431$ 407 


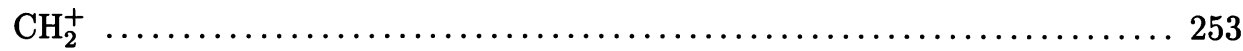

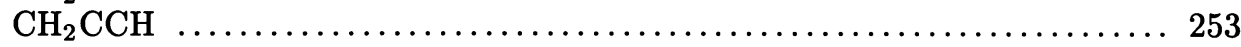

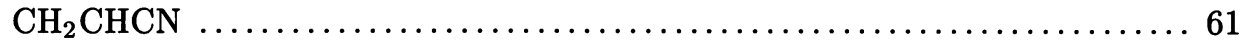

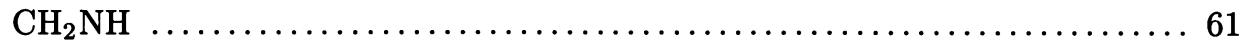

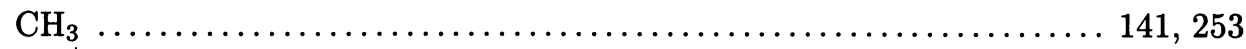

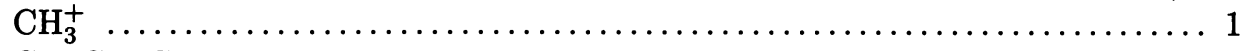

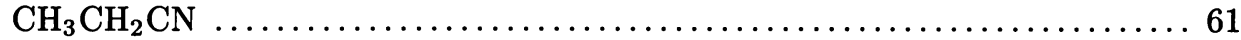

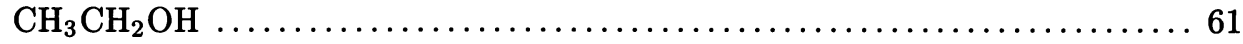

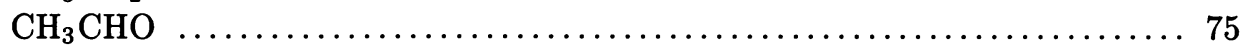

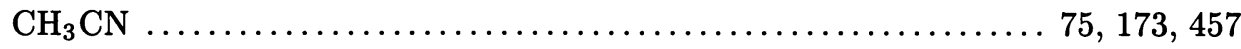

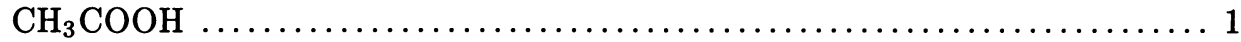

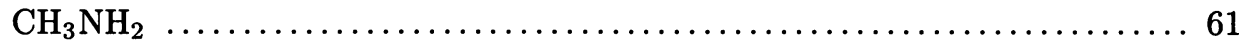

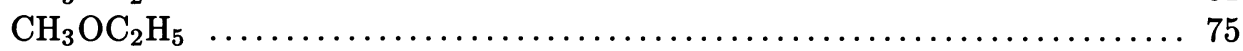

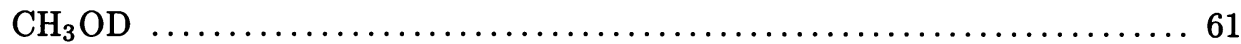

$\mathrm{CH}_{3} \mathrm{OH} \ldots \ldots \ldots \ldots \ldots \ldots \ldots \ldots \ldots . \ldots \ldots \ldots, 45,61,75,103,163,183,219,331,343$

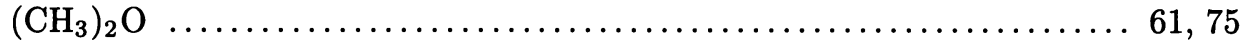

$\mathrm{CH}_{4} \ldots \ldots \ldots \ldots \ldots \ldots \ldots 13,31,45,75,103,219,253,281,321,331,441,469$

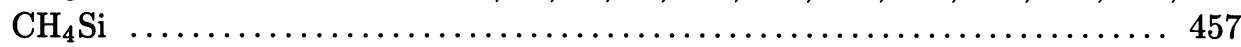

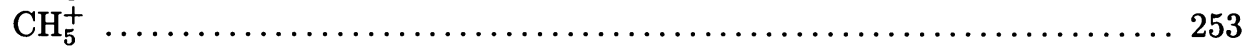

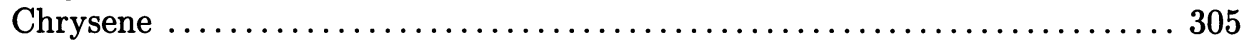

CN $\ldots \ldots \ldots \ldots \ldots \ldots \ldots \ldots 1,13,61,103,141,253,407,421,441,457,477$

CO . 1, 13, 19, 31, 45, 103, 113, 129, 141, 173, 183, 205, 219, 271, 321, 331, 343, $373,389,393,397,401,407,421,431,441,457,477,469,489,501,515,525$

${ }^{13} \mathrm{CO}$

$31,103,129$

$\mathrm{C}^{18} \mathrm{O}$

$31,103,183,193$

$\mathrm{CO}^{+}$

$1,141,477$

$\mathrm{CO}_{2}$

$1,31,45,103,219,271,331,343,373,401$

$\mathrm{CO}_{2}^{+}$

219

Coronene

305,469

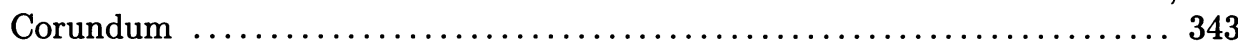

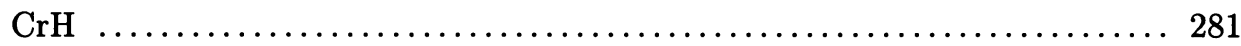

CS $\ldots \ldots \ldots \ldots 19,31,75,141,183,193,205,219,421,441,457,477,489,515$

$\mathrm{CS}_{2}$

205,219

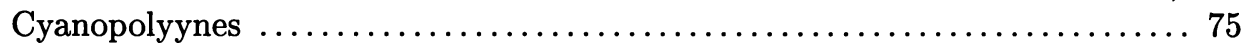

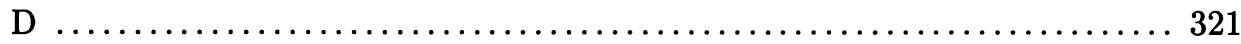

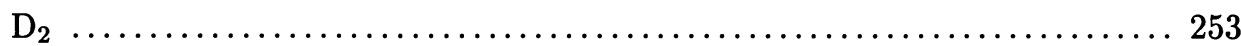

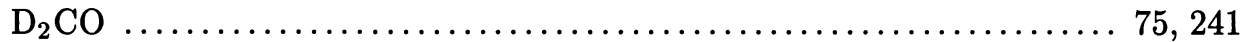

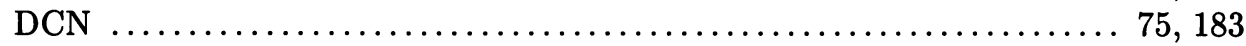

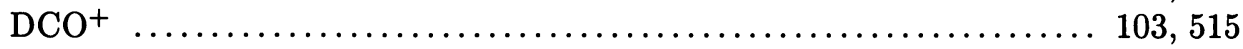

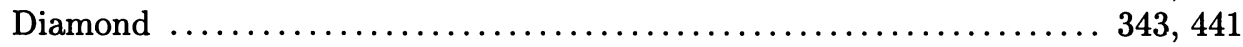

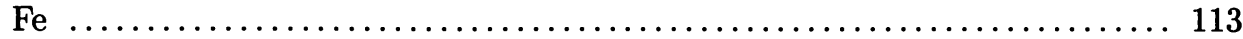

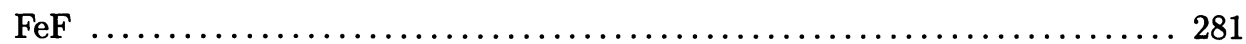

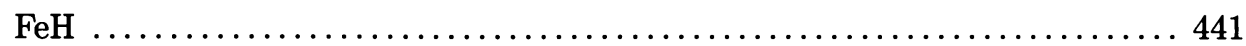

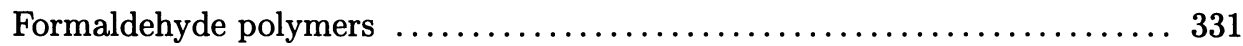

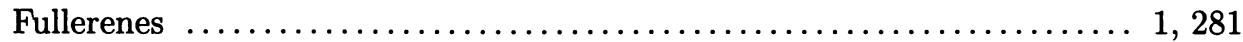

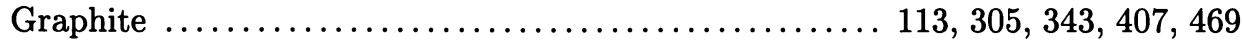

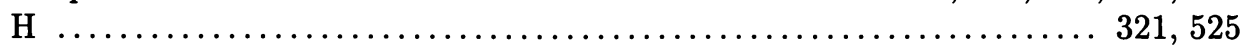


$\mathrm{H}^{-}$

$\mathrm{H}_{2} \ldots \ldots \ldots 1,13,45,141,183,253,271,321,331,373,431,441,469,477,525$

$\mathrm{H}_{2}^{+}$

$\mathrm{H}_{2} \mathrm{C}_{6}$

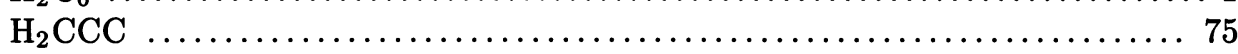

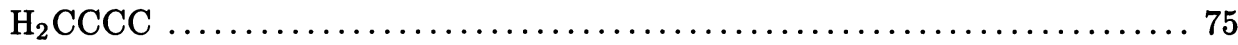

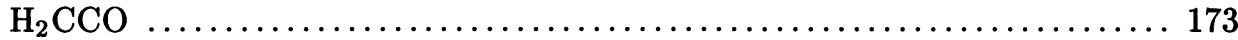

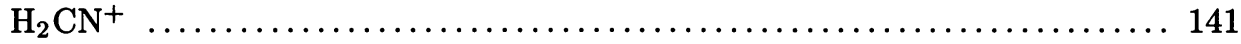

$\mathrm{H}_{2} \mathrm{CO} \ldots 19,31,45,61,75,103,163,173,183,193,219,241,331,421,515,525$

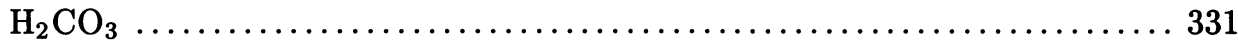

$\mathrm{H}_{2} \mathrm{COH}^{+}$

103,173

$\mathrm{H}_{2} \mathrm{CS}$

$1,13,45,75,103,113,141,163,173,205,219,271$

$\mathrm{H}_{2} \mathrm{O}$

$281,295,321,331,343,367,373,385,389,393,397,401,407,441,457,505$

$\mathrm{H}_{2} \mathrm{O}_{2}$

45,331

$\mathrm{H}_{2} \mathrm{~S}$

$61,75,103,173,219,421$

$\mathrm{H}_{3}^{+}$ 141

$\mathrm{H}_{3} \mathrm{O}^{+}$

$1,141,219$

HAC 343

$\mathrm{HBr}$ 253

$\mathrm{H}\left(\mathrm{C}_{2}\right)_{n} \mathrm{CN}$ 253

$\mathrm{H}\left(\mathrm{C}_{2}\right)_{n} \mathrm{H}$ 253

$\mathrm{HC}_{3} \mathrm{~N}$ $31,61,75,173,219,457,477$

$\mathrm{HC}_{5} \mathrm{~N}$

75,457

$\mathrm{HC}_{7} \mathrm{~N}$

75,457

$\mathrm{HC}_{9} \mathrm{~N}$

$\mathrm{HC}_{11} \mathrm{~N}$

$\mathrm{HC}_{13} \mathrm{~N}$

$\mathrm{HC}_{n} \mathrm{H}$

$\mathrm{HC}_{n} \mathrm{H}^{+}$

$\mathrm{HC}_{n} \mathrm{~N}^{+}$

$\mathrm{HCCN}$

$\mathrm{HCl}$

407,441

HCN . 31, 61, 103, 141, 183, 205, 331, 389, 397, 421, 441, 457, 477, 489, 515, 525

$\mathrm{HCN}^{+}$

$\mathrm{HCO}$

$\mathrm{HCO}^{+}$ $1,19,31,103,141,183,193,421,431,457,477,489,515,525$

$\mathrm{H}^{13} \mathrm{CO}^{+}$ 103

$\mathrm{HCONH}_{2}$

$\mathrm{HCOO}^{-}$

$\mathrm{HCOOCH}_{3}$

$31,61,75$

$\mathrm{HCOOH}$ 45,331

HDCO

HDO $31,75,183,219,271$

$\mathrm{He}^{+}$

$\mathrm{HeH}^{+}$ 
HNC

$61,219,421,477,489,515,525$

HNCO

$31,61,331$

HOCN

331

Hydrated silicates 343

$\mathrm{KCl}$

281,457

$\mathrm{LaO}$ 441

Magnetite 343

Metallic iron 343

$\mathrm{Mg}^{+}$ 525

$\mathrm{Mg}^{7+}$ 373

$\mathrm{MgCN}$ 457

$\mathrm{MgF}$ 457

$\mathrm{MgH}$ 441

$\mathrm{MgNC}$ 457

$\mathrm{N}$

$\mathrm{N}^{+}$ 253,271

$\mathrm{N}^{2+}$ 389

$\mathrm{N}_{2}$ 389

$\mathrm{N}_{2} \mathrm{H}^{+}$ $45,219,321,331$

$\mathrm{N}_{2} \mathrm{H}_{2}$ $103,183,193,477,525$

$\mathrm{N}_{2} \mathrm{H}_{4}$ 331

$\mathrm{NaCl}$ 331

$\mathrm{NC}_{n} \mathrm{~N}^{+}$ 281,457

$\mathrm{Ne}^{+}$ 287

$\mathrm{Ne}^{2+}$ 373

$\mathrm{Ne}^{4+}$ 367,373

$\mathrm{Ne}^{5+}$ 373

$\mathrm{NH}$ 373

$\mathrm{NH}_{2}$ $219,271,407,441$

$\mathrm{NH}_{2} \mathrm{CHO}$ 219

$\mathrm{NH}_{2} \mathrm{CN}$ 61 $\mathrm{NH}_{2} \mathrm{D}$ 61,75

$\mathrm{NH}_{3}$ $13,19,61,75,163,205,219,331$

NO 141,253

$\mathrm{NO}^{+}$ 407

$\mathrm{O}$

$\mathrm{O}^{+}$ $113,141,173,253,271,389,393,505$

$\mathrm{O}^{2+}$ 525

$\mathrm{O}^{3+}$ 389

$\mathrm{O}_{2}$ 373

$\mathrm{O}_{3}$

$\mathrm{O}_{4}$ $13,45,103,113,141,253,331$

$\mathrm{OCN}^{-}$ 13,331
$\ldots . .13$ OCS 331

$\mathrm{OH}$ 331 $45,103,205,219,331$

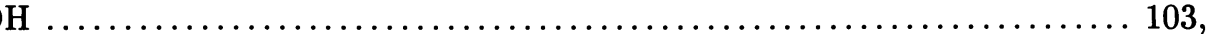
Olivine $113,141,163,219,241,253,271,373,389,393,407,431,441,457,477,505$ 


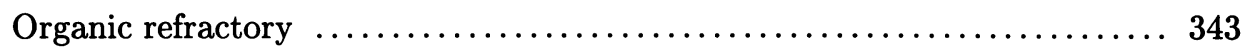

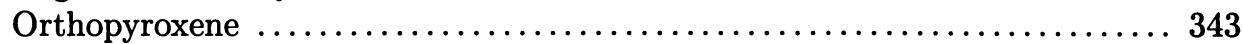

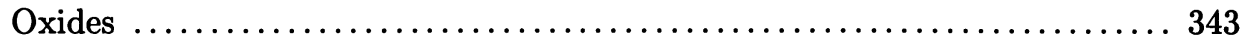

PAH $\ldots \ldots \ldots \ldots \ldots \ldots \ldots \ldots \ldots \ldots . \ldots \ldots . .21,219,281,305,343,357,373,441,469$

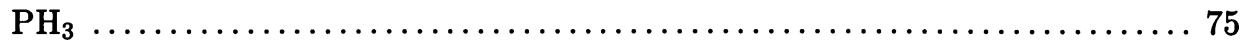

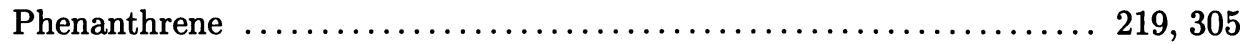

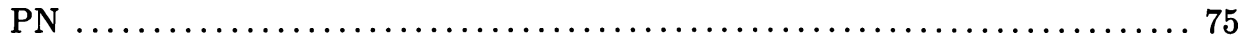

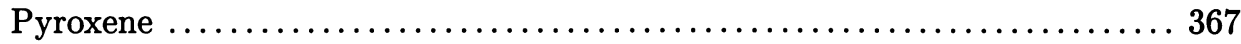

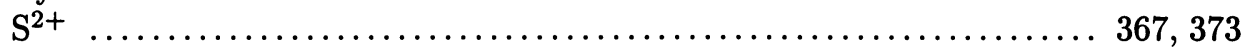

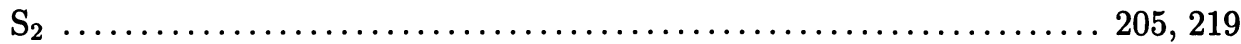

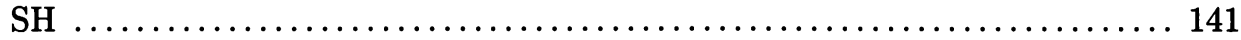

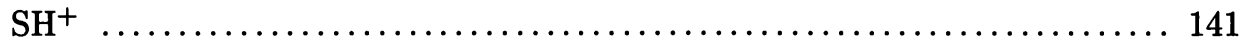

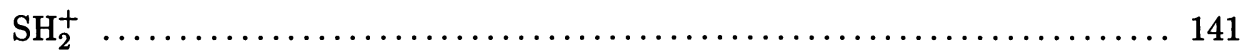

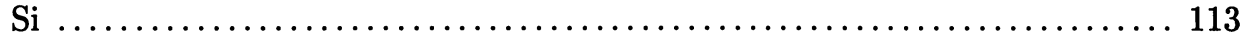

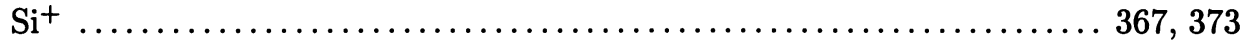

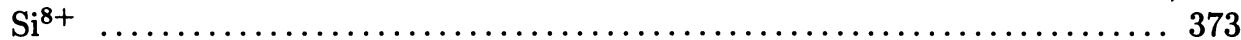

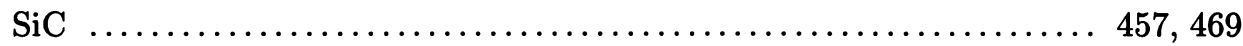

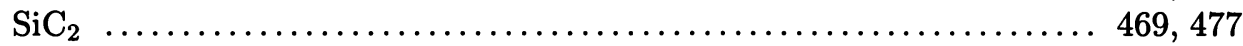

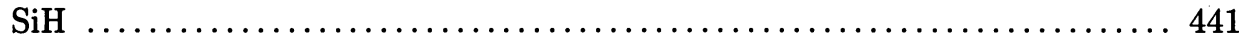

$\mathrm{SiH}_{4} \ldots \ldots \ldots \ldots \ldots \ldots \ldots \ldots \ldots \ldots \ldots \ldots \ldots \ldots \ldots \ldots \ldots \ldots \ldots \ldots \ldots \ldots \ldots \ldots \ldots \ldots \ldots, 113$

$\mathrm{SiO} \ldots \ldots \ldots \ldots \ldots \ldots \ldots 19,31,61,75,103,113,163,281,441,457,469,477$

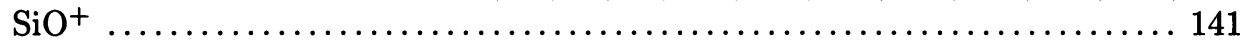

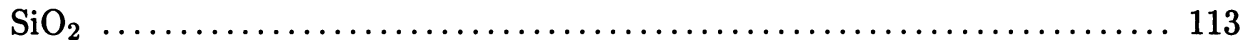

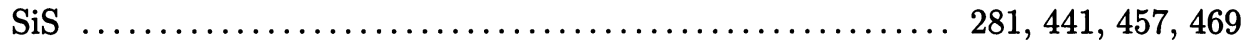

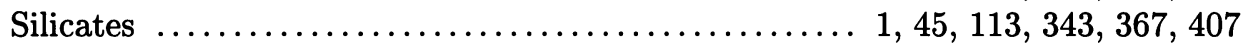

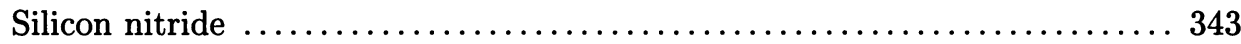

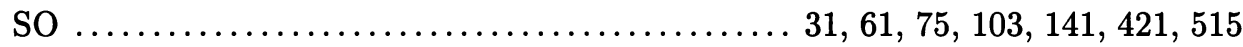

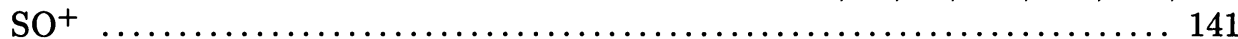

$\mathrm{SO}_{2} \ldots \ldots \ldots \ldots \ldots \ldots \ldots \ldots \ldots \ldots \ldots \ldots \ldots \ldots \ldots \ldots \ldots \ldots, 61,75,103,141,219,331$

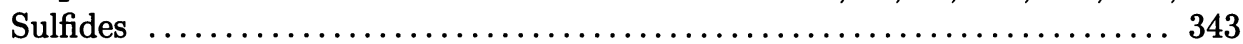

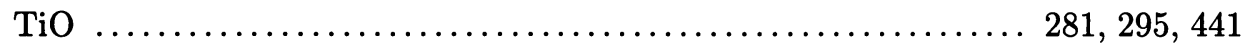

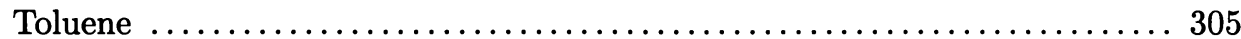

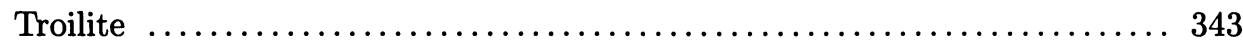

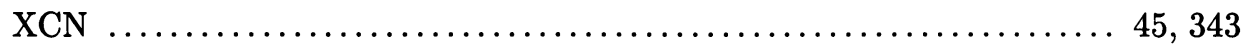

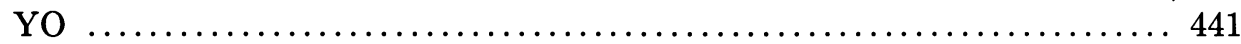

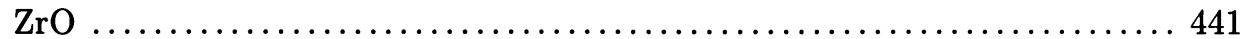

